\title{
Toroidal photonic metamaterial
}

\author{
V. Savinov ${ }^{1, *}$, V. A. Fedotov ${ }^{1}$, W. T. Chen ${ }^{3}$, Y. W. Huang ${ }^{3}$, D. P. Tsai ${ }^{3}$, D. B. Burckel ${ }^{2}$, \\ I. Brener ${ }^{2}$ and N. I. Zheludev ${ }^{1}$ \\ ${ }^{1}$ Optoelectronics Research Centre \& Centre for Nanostructured Photonic Metamaterials, University of Southampton, SO17 1BJ, UK \\ ${ }^{2}$ Sandia National Laboratories, New Mexico, NM 87185, USA \\ ${ }^{3}$ Department of Physics, National Taiwan University, Taipei 10617, Taiwan \\ *vs1106@orc.soton.ac.uk
}

\begin{abstract}
We present the first design of a photonic metamaterial demonstrating dominant toroidal dipolar response in the infrared part of the spectrum.

OCIS Codes: (160.3918) Metamaterials; (160.4670) Optical materials
\end{abstract}

We report on the development and fabrication attempt of toroidal metamaterials operating in the near-to-far IR spectral range. The toroidal metamaterials is a recently demonstrated novel class of artificial media with the resonant macroscopic response determined by a peculiar and previously disputed fundamental type of microscopic multipolar excitations, the toroidal dipole. Taking into account limitations of the most suited fabrication techniques (namely Sandia's projection lithography SAMPL and NTU's e-beam lithography with precise alignment technique) and using realistic data for the optical properties of the constituent dielectrics and metals, we have come up with a novel design of such metamaterials capable of producing dominant toroidal response at IR frequencies.

Toroidal dipole is a fundamental electromagnetic excitation, which cannot be represented in terms of the existing standard multipole expansion. Toroidal dipole is associated with toroidal current configurations and was first considered by Zel'dovich back in 1957 [1], but only some 20 years ago become the subject of growing interest owing to its peculiar electromagnetic properties. For example, electromagnetic interactions with toroidal currents were predicted to disobey such widely accepted principle as the action-reaction equality. Toroidal currents can also form charge-current configurations generating vector potential fields in the absence of radiated electromagnetic waves. Although toroidal dipole is held responsible for parity violation in nuclear and particle physics, the effects associated with toroidal excitations in naturally available materials are extremely weak and no direct evidence of their importance for classical electrodynamics has been reported until recently [2].

Toroidal dipole, unlike conventional electric and magnetic dipoles, couples to electromagnetic fields very weakly and is therefore much more susceptible to dissipative losses making the demonstration of toroidal excitation at optical frequencies a very challenging task. By careful multipole engineering we have been able to come up with the design of a toroidal metamaterial with a strong resonance in the infrared, where all the standard multipoles are substantially suppressed rendering toroidal dipolar scattering the dominant mechanism of the metamaterial optical response. A single meta-atom of our metamaterial is shown on Fig. 1a.
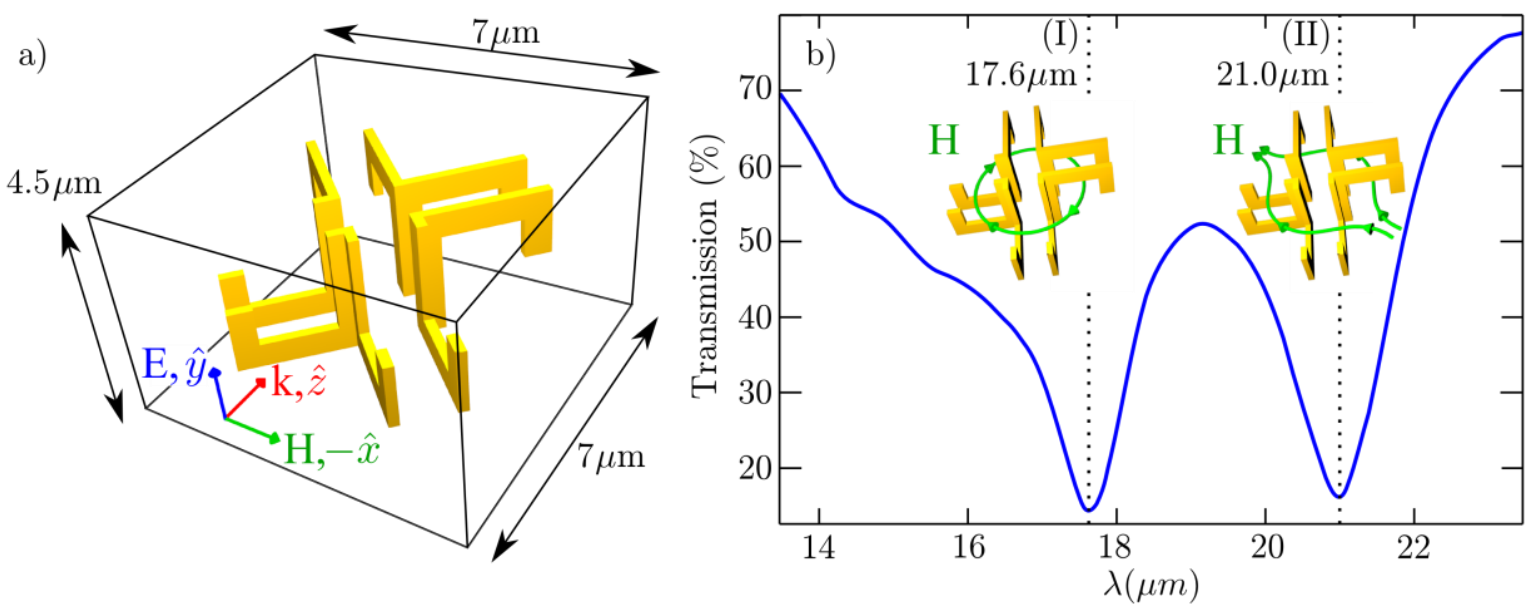

Fig 1: Toroidal photonic metamaterial. a) The design of the single unit cell. The meta-atom is composed from four pairs of gold split ring resonators arranged on two mutually orthogonal planes, immersed in SU8 dielectric. The metamaterial is formed by translating the unit cell along X-and Y-directions. b) Simulated transmission of the metamaterial for the case of realistic material parameters of gold and dielectric host. The metamaterial exhibits two resonances at $17.6 \mu \mathrm{m}$ (I) and $21.0 \mu \mathrm{m}$ (II). The insets show artistic impressions of the local magnetic field configurations at the two resonances. 
Four pairs of gold split ring resonators are immersed in low-loss dielectric (SU8) and reside on two mutually orthogonal planes. The full size of the unit cell is $7 \mu \mathrm{m} \times 4.5 \mu \mathrm{m} \times 7 \mu \mathrm{m}$ and metamaterial is created by translating the unit cell along X-and Y-direction. The metamaterial is driven by free-space electromagnetic radiation with polarization as shown on Fig. 1a. Fig. 1b shows the simulated transmission of the designed metamaterial for the case of realistic material constants of gold and dielectric host. Two clear resonances are observable at $17.6 \mu \mathrm{m}$ (I) and $21 \mu \mathrm{m}$ (II). The longer-wavelength resonance (II) corresponds to magnetic dipoles of all four pairs of split rings of the meta-molecule oscillating in phase, so the net response of the meta-atom is dominated by the magnetic dipole in X-direction. The short-wavelength resonance (I) corresponds to magnetic dipoles of the front two split ring resonator pairs oscillating in anti-phase with the rear two split ring resonator pairs. The magnetic field, thus, oscillates in a closed contour at each meta-molecule. This mode corresponds to the toroidal dipole.

Using the distribution of current density in the meta-molecule obtained from solution of 3D Maxwell equations we computed the electromagnetic power scattered by all multipoles in the multipole expansion of meta-molecule on Fig. 1a when driven by normally incident free-space radiation. Four leading multipoles are shown on Fig. 2. One can clearly see that at the short-wavelength resonance (I) the response of our metamaterial is dominated by the toroidal dipolar scattering.

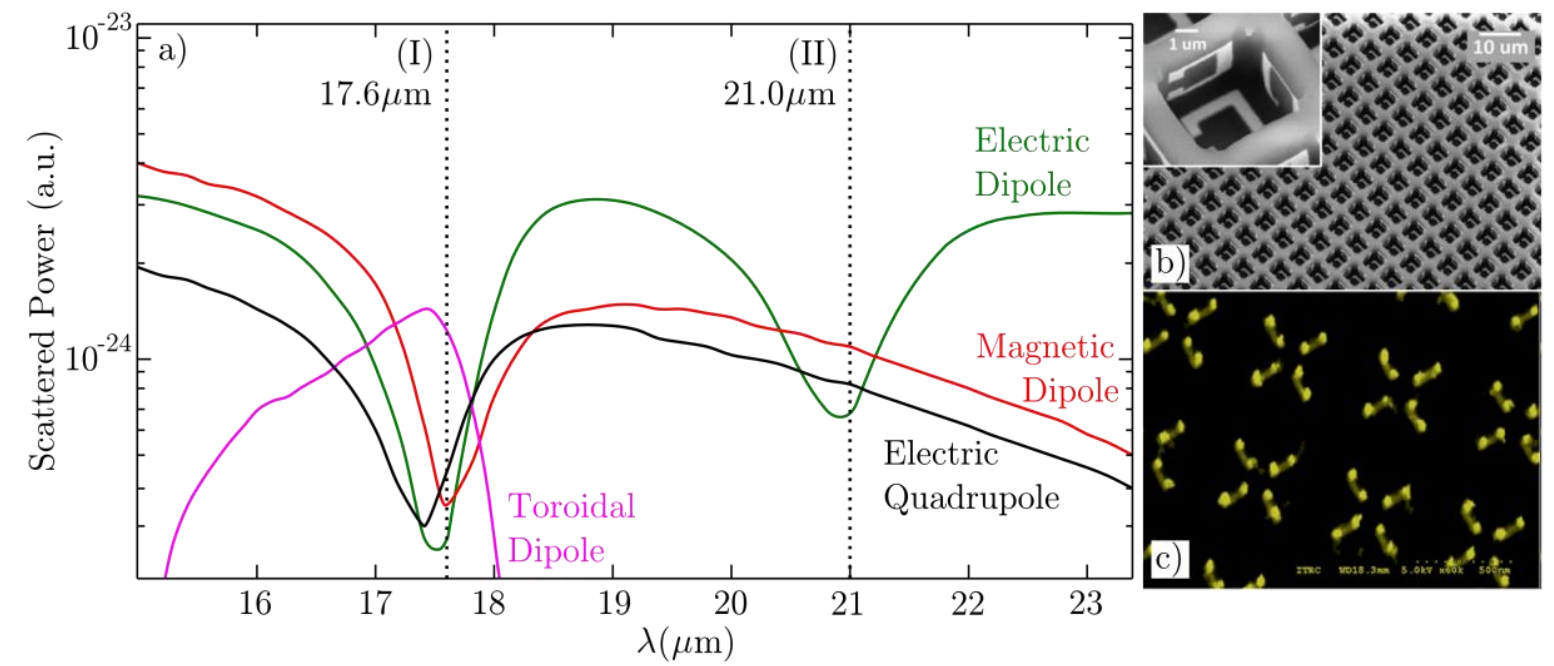

Fig 2: a) The power scattered by five leading multipoles computed from current density distribution inside the unit cell of the metamaterial. The two resonances shown on Fig. 1 b) are clearly visible, with the short-wavelength resonance (I) being dominated by toroidal dipole scattering and long-wavelength resonance (II) being dominated by magnetic dipole scattering. b) SEM image of an array of split-ring based 3D meta-molecules fabricated using projection lithography SAMPL. c) SEM image of an array of 3D toroidal meta-molecules fabricated using e-beam lithography with high precision alignment.

We have also investigated the possibility of enhancing toroidal response further by incorporating gain into the fabric of the toroidal metamaterial. Importantly, our metamaterial design takes into account the limitations of the existing 3D nanofabrication techniques, such as Sandia's projection lithography SAMPL (Fig. 2b) and NTU's e-beam lithography with high precision alignment technique (Fig. 2c). The results of the first fabrication attempts will be reported.

In conclusion, we report the first results on the design and development of a novel type of 3D photonic metamaterials with the dominant toroidal dipolar response in the IR part of the spectrum.

Sandia National Laboratories is a multi-program laboratory managed and operated by Sandia Corporation, a wholly owned subsidiary of Lockheed Martin Corporation, for the U.S. Department of Energy's National Nuclear Security Administration under contract DE-AC04-94AL85000.

[1] Ia .B. Zel'dovich, "Electromagnetic interaction with parity violation,” Sov. Phys. JETP 6, 1184-1186 (1958).

[2] T. Kaelberer, V. A. Fedotov, N. Papasimakis, D. P. Tsai, N. I. Zheludev, “Toroidal Dipolar Response in a Metamaterial,” Science 330, $1510-1512(2010)$. 